\title{
CT ANGIOGRAPHIC STUDY OF THE ROLE OF BOTH WILLIS CIRCLE AND VERTEBRAL ARTERIES DURING SELECTIVE CEREBRAL PERFUSION - A STUDY IN 105 PATIENTS
}

\author{
Papantchev V. ${ }^{1,4}$, V. Stoinova ${ }^{2}$, D. Todorova-Papantcheva ${ }^{3}$, V. Groudeva ${ }^{2}$, A. Paloff ${ }^{4}$, \\ D. Hinova-Palova ${ }^{4}$, S. Hristov ${ }^{4}$, A. Aleksandrov ${ }^{5}$, M. Goshev $^{5}$, D. Nikolov ${ }^{5}$, \\ D. Petkov ${ }^{1}$, G. Nachev ${ }^{1}$, W. Ovtscharoff ${ }^{4}$ \\ ${ }^{1}$ Department of Cardiac Surgery, ${ }^{2}$ Section of Visual Diagnostics, St. Ekaterina University Hospital \\ of Sofia, Medical University of Sofia, ${ }^{3}$ Department of General, Experimental and Genetic \\ Psychology, Kliment Ohridski University of Sofia, ${ }^{4}$ Department of Anatomy and Histology, \\ Medical University of Sofia, ${ }^{5}$ Department of Forensic Medicine, Medical University of Sofia
}

\section{ABSTRACT}

Unilateral selective cerebral perfusion (SCP) is a method for cerebral protection in aortic arch surgery. However, variations of the circle of Willis $(\mathrm{CoW})$ could vitiate its protective effect. The aim of our present work was to prospectively analyze variations of $\mathrm{CoW}$ and vertebral arteries using $\mathrm{CT}$ angiography. From January, 2008 to July, 2008, a total of 105 consecutive patients underwent CT-angiography of the CoW in the Division of Radiology, St. Ekaterina University Hospital of Sofia. There were at least six CoW configurations that could lead to significant hypoperfusion during unilateral SCP: i) type IA - hypoplasia or absence of left posterior communicating artery (PComA) (in 41,9\% of the patients); ii) type IB - hypoplasia or absence of anterior communicating artery (AComA) (in 1,9\%); iii) type IIA - hypoplasia or absence of both left PComA and AComA (in 6,67\%); iv) type IIB - hypoplasia or absence of left P1 or right vertebral artery (VA) (in 6,67\%); v) type III - hypoplasia or absence of right A1 (in 8,57\%), and vi) type IV - hypoplasia or absence of both right A1 and right VA or both right $A 1$ and left $P C o m A$ (in $0,95 \%$ ). All these types were present in a $66,67 \%$ of all the examined patients. Our present study showed that $\mathrm{CoW}$ variations are presented in significant number of patients. These results support the need of extensive preoperative examination and meticulous intraoperative monitoring of cerebral perfusion during unilateral SCP.

Key words: cerebral protection, selective cerebral perfusion, aortic surgery, Willis circle variations, stroke

\section{INTRODUCTION}

Cerebral protection $(\mathrm{CP})$ is a key issue during aortic arch surgery $(10,11)$. This is required in patients with DeBakey type I acute or chronic aortic dissections (11). The major goal of CP is to guarantee the optimal ratio between the blood supply and the metabolic demands of the brain (11). Selective cerebral perfusion (SCP) is a method for CP widely used in aortic surgery. It could be subdivided into unilateral SCP performed with arterial canulation of brachiocephalic trunk or some of its branches, and bilateral SCP performed with canulation of both brachiocephalic trunk and left common carotid artery $(10,11)$. During the unilateral SCP, brain receives blood only through the right common carotid artery and right vertebral artery (VA). The

\footnotetext{
Address for correspondence

V. Papantchev, Dept. of Cardiac Surgery,

St. Ekaterina University Hospital of Sofia,

Medical University of Sofia,

1 Georgi Sofiyski Str., 1431 Sofia, Bulgaria

e-mail: vassil_papanchev@yahoo.com
}

assumption for protective effect of unilateral SCP is based on the understanding that collateral circulation, mainly through the circle of Willis (CoW), is sufficient to maintain adequate perfusion in the contralateral (left) hemisphere (Fig. 1). According to the literature data, some $\mathrm{CoW}$ variations exist in at least $50 \%$ of the people $(15,16)$.

The aim of our present work was to study the variations of CoW and VAs by means of CT angiography. Some of our data were already presented elsewhere (17).

\section{MATERIAL AND METHODS}

\section{Patients}

From January $4^{\text {th }}, 2008$ to July $30^{\text {th }}, 2008$, a total of 105 consecutive patients, 52 male and 53 female, with average age of 61,9 years (range, 18-85 years) underwent CT-angiography of the CoW in the Division of Radiology, St. Ekaterina University Hospital of Sofia. The vast majority of these patients suffered from a neurological disease 

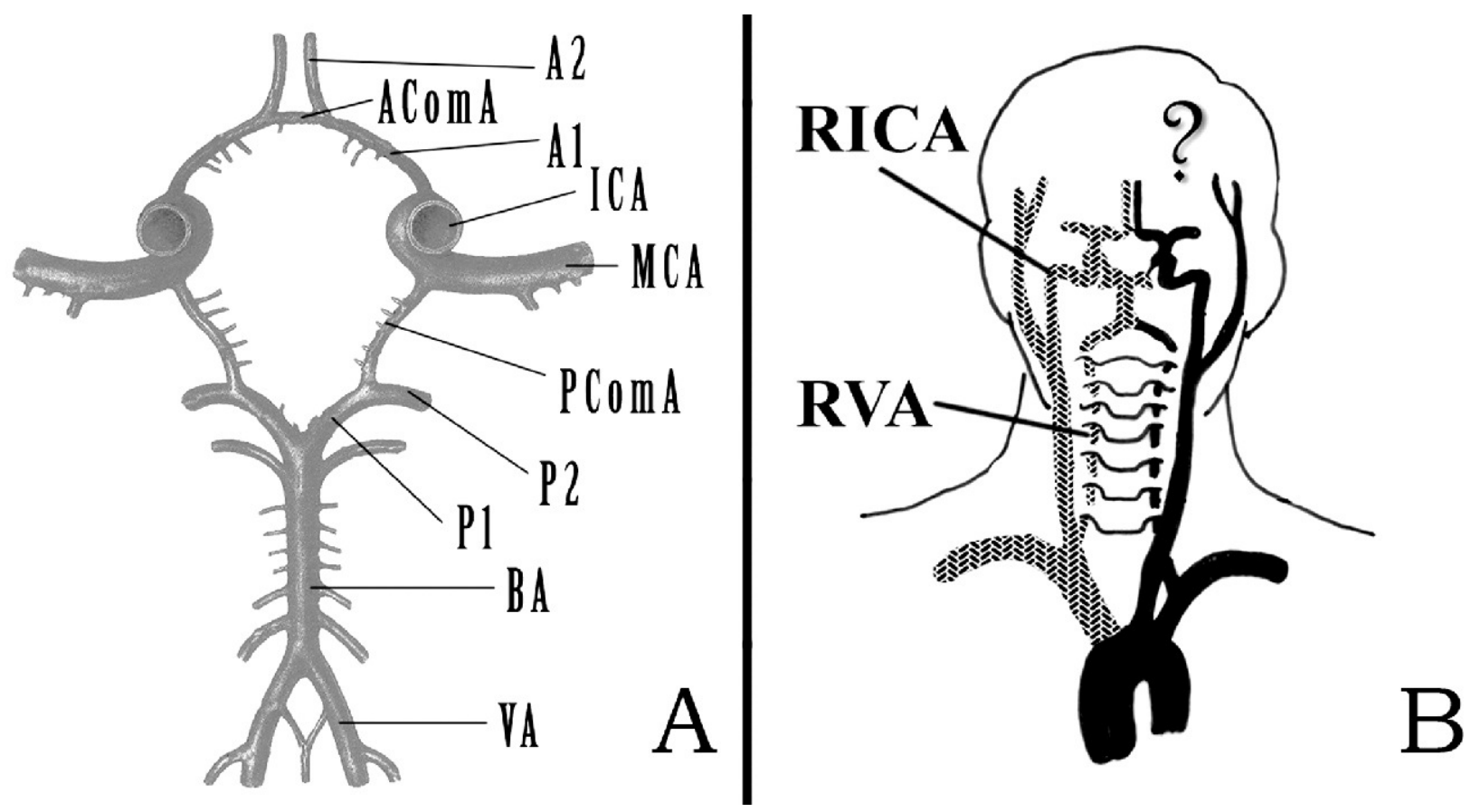

Fig. 1 A - normal morphology and segments of CoW; 1 B - hemodynamics during the unilateral SCP: hatched vessels with direct perfusion (right internal carotid artery and right vertebral artery)

before the examination. We prospectively collected the examinations data.

\section{CT angiography}

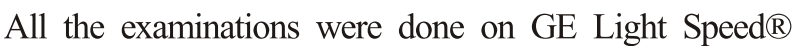
16-slice computer tomography (General Electric, USA) during the arterial phase after i.v. administration of $60 \mathrm{~mL}$ of iodine-based contrast medium (Iomeron ${ }^{\circledR} 400 \mathrm{mg} / \mathrm{mL}$; Patheon Italia S.p.A, Italy) with injection speed of 4 $\mathrm{mL} / \mathrm{s}$. The slice thickness was $1,25 \mathrm{~mm}$. After examination all the axial, multiplanar and $3 \mathrm{D}$ reconstruction images were analyzed on work station Advantage.

\section{RESULTS}

Table 1. Comparison of variations of CoW, significant during unilateral SCP

\begin{tabular}{|c|c|c|c|}
\hline CoW type & $\begin{array}{l}\text { Papantchev } \\
\text { et al., (4) }\end{array}$ & $\begin{array}{l}\text { Papantchev } \\
\text { et al., (3) }\end{array}$ & Present study \\
\hline $1 \mathrm{~A}$ & $26,6 \%(22 / 83)$ & $27,3 \%(27 / 99)$ & $41,9 \%(15 / 105)$ \\
\hline 1B & $2,4 \%(2 / 83)$ & $0 \%(0 / 99)$ & $1,9 \%(0 / 105)$ \\
\hline $2 \mathrm{~A}$ & $3,6 \%(3 / 83)$ & $3,1 \%(3 / 99)$ & $6,67 \%(3 / 105)$ \\
\hline $2 \mathrm{~B}$ & $7,2 \%(6 / 83)$ & $9,1 \%(9 / 99)$ & $6,67 \%(0 / 105)$ \\
\hline 3 & $7,2 \%(6 / 83)$ & $3,1 \%(3 / 99)$ & $8,57 \%(2 / 105)$ \\
\hline 4 & $1,2 \%(1 / 83)$ & $0 \%(0 / 99)$ & $0,95 \%(1 / 105)$ \\
\hline total & $48,2 \%(40 / 83)$ & $42,4 \%(42 / 99)$ & $\begin{array}{l}66,67 \% \\
(21 / 105)\end{array}$ \\
\hline
\end{tabular}

CoW variations which could cause significant hypoperfusion during the unilateral SCP were observed during this study. For their classification, we used classification of $\mathrm{CoW}$ variations proposed earlier in the literature available (15-17). This classification divided CoW variations in six configurations (Fig. 2) based on the most probable zone of hypoperfusion (Fig. 3) as defined according to both hemodynamics during the unilateral SCP and Poiseulles-Hagen's law (15-17). All the six CoW types were observed in this study, namely:

i. type IA - hypoplasia or absence of left posterior communicating artery (PComA) (in $41,9 \%$ of the patients) (Fig. 2A, 2B and 2C);

ii. type IB - hypoplasia or absence of anterior communicating artery (AComA) (in 1,9\%); (Fig. 2D, $2 \mathrm{E}$ and $2 \mathrm{~F}$ );

iii. type IIA - hypoplasia or absence of both left PComA and AComA (in 6,67\%) (Fig. 2G, 2H and 2I);

iv. type IIB - hypoplasia or absence of left P1 or right vertebral artery (VA) (in 6,67\%) (Fig. 2J, 2K and 2L);

v. type III - hypoplasia or absence of right A1 (in 8,57\%) (Fig. 2M, 2N and 2O), and

vi. type IV - hypoplasia or absence of both right A1 and right VA or both right $\mathrm{A} 1$ and left PComA (in 0,95\%). (Fig. 2P, 2Q and 2R).

The configuration of type IV CoW is described for the first time in the literature by us (17).

These types were present in $66,67 \%$ of the examined patients. Besides we established that many patients have significant stenosis of carotid, vertebral or other cerebral artery. 


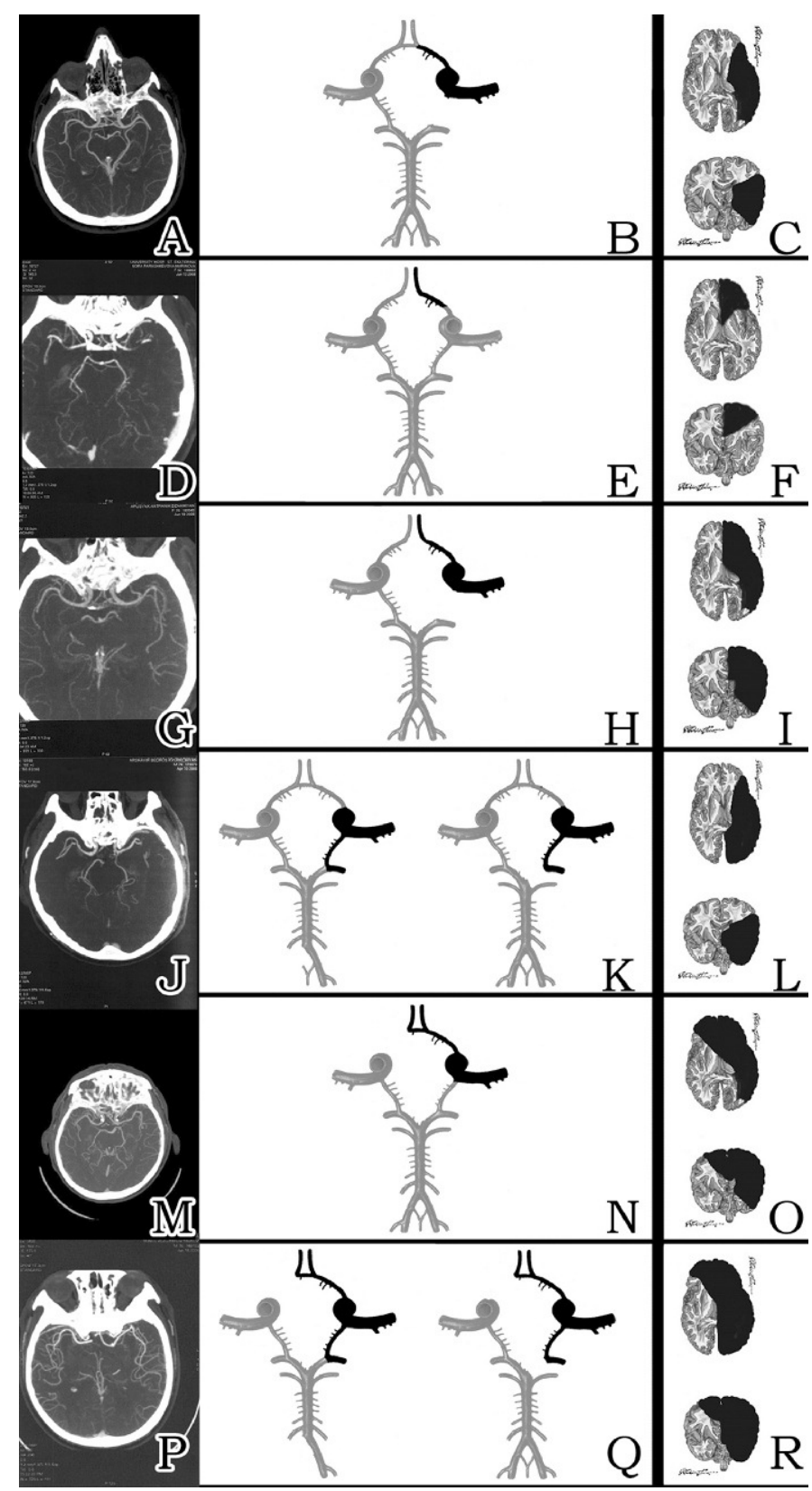

Fig. 2. CoW variations

A. CT of CoW type $1 A$ with hypoplasia of left PComA

B. Scheme of CoW type 1A. Left median cerebral artery (MCA) is the zone of hypoperfusion (shown in black)

C. Cerebral cortex at risk of hypoperfusion during the unilateral SCP if CoW type $1 \mathrm{~A}$ is present

D. CT of CoW type $1 B$ with hypoplasia of AComA

E. Scheme of CoW type 1B. Left anterior cerebral artery (ACA) is the zone of hypoperfusion (shown in black)

$F$. Cerebral cortex at risk of hypoperfusion during the unilateral SCP if CoW type $1 B$ is present

G. CT of CoW type $2 A$ with hypoplasia of both left PComA and AcomA

$H$. Scheme of CoW type 2A. Both left MCA and left ACA are at risk of hypoperfusion (shown in black)

I. Cerebral cortex at risk of hypoperfusion during the unilateral SCP if CoW type $2 A$ is present

$J$. CT of CoW type $2 B$ with hypoplasia of right P1 segment of left posterior cerebral artery (PCA)

$K$. Scheme of CoW type 2B. Both left MCA and left PCA at rish of hypoperfusion (shown in black)

L. Cerebral cortex at risk of hypoperfusion during the unilateral SCP if CoW type $2 B$ is present

M. CT of CoW type 3 with hypoplasia of Al segment of right ACA

N. Scheme of CoW type 3. Both ACA and left MCA are at risk of hypoperfusion (shown in black)

$O$. Cerebral cortex at risk of hypoperfusion during unilateral SCP if CoW type 3 is present

P. CT of CoW type 4 with hypoplasia of both left $P 1$ and right A1

Q. Scheme of CoW type 4. Four vessels are at risk of hypoperfusion, namely both ACA, left MCA and left PCA (shown in black)

R. Cerebral cortex at risk of hypoperfusion during unilateral SCP if CoW type 4 is present 


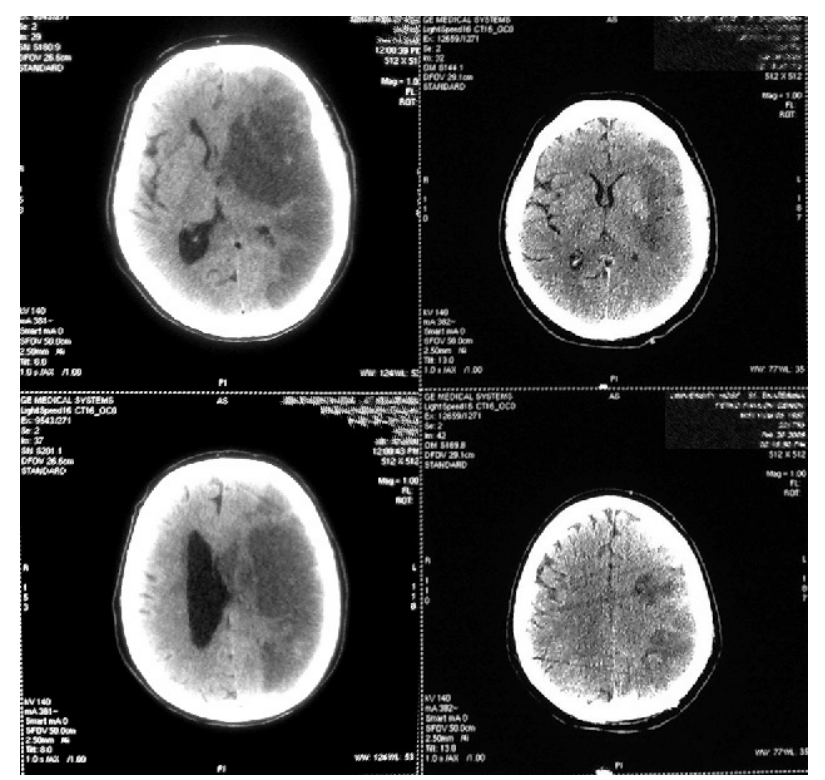

Fig. 3. Clinical role of CoW variations

A. Postoperative stroke in a patient with CoW type $1 \mathrm{Al}$

$B$. Postoperative stroke in a patient with CoW type $2 B$

\section{DISCUSSION}

According to the literature available, $\mathrm{CoW}$ variations are present in at least $50 \%$ of the population $(1,2,4-9,12-20)$. Major disadvantage of most studies is, however, that they examine each segment of the circle separately $(1,2,7,14,20)$. It is noteworthy that the variations often affect more than one segment of CoW $(4-6,8,9,12,13,15-19)$. As it was mentioned above, the hemodynamics during the unilateral SCP is unique, i.e. brain receives blood only through the right common carotid and right VA. For this reason, CoW variations could lead to a significant reduction of perfusion in certain brain areas during the unilateral SCP and thus to vitiate its protective effect (15-17).

Up to present, 13 publications studied CoW variations as a whole $(3-6,8,9,12,13,15-19)$. Only five of them, three of which being published by our group raise the question about the role of CoW variation during the unilateral SCP $(13,15-17,19)$. Unfortunately, the data from these five studies couldn't be compared neither between each other, nor with the rest 8 studies $(3-6,8,9,12,13,15-19)$ for the following reasons:

First, seven of these studies $(4-6,8,9,12,18)$ classify CoW variations without paying attention of their lateralization. Since the hemodynamics during the unilateral SCP is unique, the left-right lateralization is of crucial importance (16).

Second, in 9 of the studies, including those of Merkkola et al. (13) and Urbanski et al. (19), there are no data about VAs at all $(4-6,8,9,12,13,18,19)$. This fact is not a surprise as VAs are not a part of CoW per se, however, their variations could present with a crucial importance during the unilateral SCP (16).
Third, there are deviations in variations' classification and definitions of 'hypoplastic'. Thus any arteries with diameter under $0,5 \mathrm{~cm}$ are considered hypoplastic (13), while in most previous publications, the size of $1 \mathrm{~mm}$ was used as a threshold (4-6,8,9,12,15-18). Other authors do not fix any size when defining the hypoplastic artery (19).

And, finally, Dadmehr et al. (3) published their work in Arabic and thus we failed to use it for comparison.

For all these reasons, we could compare our results with our previous three publications (15-17) only. The first study was theoretical and used illustrations, so its results should be interpreted with caution (15). The second study use autopsy material (3), while the third was available as an abstract only and thus reported briefly our findings (17).

Our present data and those from the first two studies are in good agreement in some aspects but seriously discrepant in other aspects (Table 1). Therefore, the most frequent variation was type $1 \mathrm{~A}$ in these three studies, however, the frequency in our present study was higher by $10 \%$. The frequency of the other types was similar to that one reported in the literature (15-17). In the present study we reported a new, type $4 \mathrm{CoW}$ configuration that was the rarest and the most severe one (Fig 2P, 2Q and 2R). Finally, the overall frequency of $\mathrm{CoW}$ variations was between $48,2 \%$ and $42,4 \%$ in previous studies $(15,16)$ but of $66,67 \%$ in the present investigation. The explanation must be sought in the examined population. Most subjects in the most recent study of ours had suffered from a neurological disease before the examination. The examinations of $\mathrm{CoW}$ in $994 \mathrm{pa}-$ tients with a previous history of neurological dysfunction revealed a normal CoW in only $21 \%$ of them (18).

\section{CONCLUSION}

Our present study shows that $\mathrm{CoW}$ variations are presented in a significant number of patients. This study supports the need of extensive preoperative examination, including CT angiography, especially in patients with preoperative neurological symptoms and meticulous intraoperative $\mathrm{CP}$ monitoring during the unilateral SCP such as NIRO, BIS, etc. Our present result confirm the superiority of bilateral over unilateral $\mathrm{SCP}$ as most $\mathrm{CoW}$ variations demonstrated in our present work are without any hemodynamic significance during the bilateral SCP. A more profound investigation with more patients is required in order to verify the clinical significance our present results.

\section{REFERENCES}

1. Adachi, B. Anatomie der Japaner. Das Arteriensystem der Japaner. Kyoto, Verlag der Kaiserlich-J apanischen Universität zu Kyoto, 1928, 111-134.

2. Alpers, B. J., R. G. Berry, R. M. Paddison. Anatomical studies of the circle of Willis in normal brain.- AMA Arch. Neurol. Psychiatry, 81, 1959, No 4, 409-418. 
3. Dadmehr, M., A. Fattahzade, S. Kamali Ardakani, F. Bateni, F. Nejat, S. M. Ghodsi, et al. An anatomical study of normal variations of circle of Willis in 132 fetus, newborn and adult.- Tehran Univ. Med. J., 67, 2009, 399-407 (in Arabic).

4. De Silva, K. R. D., R. Silva, D. Amaratunga, W. S. L. Gunasekera, R. Jayes ekera. Types of the cerebral arterial circle (circle of Willis) in a Sri Lankan population.- BMC Neurol., 11, 2011, 5. doi: 10.1186/1471-2377-11-5.

5. Eftekhar, B., M. Dadmehr, S. Ansari, M. Ghodsi, B. Nazparvar, E. Ketabchi. Are the distribution of variations of circle of Willis different in different populations? - Results of an anatomical study and review of literature.- BMC Neurol., 6, 2006, 22. doi: 10.1186/1471-2377-6-22.

6. El Khamlichi, A., M. Azouzi, F. Bellakhdar, A. Ouhcein, A. Lahlaidi. Anatomic configuration of the circle of Willis in the adult studied by injection technics. Apropos of 100 brains.Neurochirurgie, 31, 1985, No 4, 287-293 (in French).

7. Fawcett, E., J. V. Blachford. The circle of Willis: an examination of 700 specimens.- J. Anat. Physiol., 40, 1905, 63-70.

8. Fisher, C. M. The circle of Willis: anatomical variations.- Vasc. Dis., 2, 1965, 99-105.

9. Ghanbari, A., B. Rad, F. Ashrafian, H. Nasrabadi. A study of arterial variations of Willis circle in 100 human brains in East Azarbaijan, Iran.J. Med. Sci., 8, 2008, 747-750.

10. Kazui, T. Which is more appropriate as a cerebral protection method - unilateral or bilateral perfusion?Eur. J. Cardiothorac. Surg., 29, 2006, No 6, 1039-1040.

11. Kouchoukos, N., E. Blackstone, D. Doty, F. Hanley, R. Karp. Diseases of the thoracic arteries and veins.- In: Kirklin/Barratt-Boyes cardiac surgery. $3^{\text {rd }}$ ed. Churchill-Livingstone, 2003, vol. 2, 1799-1900.

12. Lazorthes, G., A. Gouaze, J. J. Santini, G. Salamon. The arterial circle of the brain (circulus arteriosus cerebri).- Anat. Clin., 1, 1979, 241-257.

13. Merkkola, P., H. Tulla, A. Ronkainen, V. Soppi, A. Oksala, T. Koivisto, et al. Incom- plete circle of Willis and right axillary artery perfusion.- Ann. Thorac. Surg., 82, 2006, No 1, 74-80.

14. Naydenov, E., V. Papantchev, K. Romansky, P. Shotekov, W. Ovtscharoff. Clinical aspects of the arterial blood supply and venous drainage of the brain.- Praemedicus, 26, 2005, No 1-2, 38-45.

15. Papantchev, V., D. Todorova, A. Paloff, D. Hinova-Palova, S. Hristov, E. Naydenov, et al. Some variations of the circle of Willis, important for brain protection in cardiac surgery - a new interpretation of the classical work of Adachi: "Anatomie der Japaner. Das Arteriensystem der Japaner'. Praemedicus, 27, 2006, No 1-2, 1-9.

16. Papantchev, V., S. Hristov, D. Todorova, E. Naydenov, A. Paloff, D. Nikolov, et al. Some variations of the circle of Willis, important for cerebral protection in aortic surgery - a study in Eastern Europeans.- Eur. J. Cardiothorac. Surg., 31, 2007, No 6, 982-989.

17. Papantchev, V., V. Stoinova, D. TodorovaPapantcheva, A. Paloff, D. Hinova-Palova, $\mathrm{S}$. Hristov, et al. Willis circle variations important for cerebral protection in aortic surgery - a CT angio study in Eastern Europeans.- Scr. Sci. Med., 43, 2011, p. 96.

18. Riggs, H. E., C. Rupp. Variation in form of circle of Willis. The relation of the variations to collateral circulation: anatomic analysis.- Arch. Neurol., 8, 1963, 8-14.

19. Urbanski, P., A. Lenos, J. C. Blume, V. Ziegler, B. Griewing, R. Schmitt, et al. Does anatomical completeness of the circle of Willis correlate with sufficient cross-perfusion during unilateral cerebral perfusion?- Eur. J. Cardiothorac. Surg., 33, 2008, No 3, 402-408.

20. Windle, B. On the arteries forming the circle of Willis.- J. Anat. Physiol., 22, 1887, 289-293.

\section{Acknowledgements}

This work has been supported under Grand 2011 program of Medical University of Sofia with Contract No 19/Project 20. 